\title{
A generalization of the Haemers-Mathon bound for near hexagons
}

\author{
Bart De Bruyn
}

May 21, 2019

\begin{abstract}
The Haemers-Mathon bound states that $t \leq s^{3}+t_{2}\left(s^{2}-s+1\right)$ for any finite regular near hexagon with parameters $\left(s, t, t_{2}\right), s \geq 2$. In this paper, we generalize this bound to arbitrary finite near hexagons with an order. The obtained inequality involves the orders of the quads through a given line.
\end{abstract}

MSC2010: 05B25, 51E12

Keywords: near hexagon, Haemers-Roos inequality, Haemers-Mathon bound, quad

\section{Introduction}

Suppose $\mathcal{S}=(\mathcal{P}, \mathcal{L}, \mathrm{I})$ is a partial linear space, i.e. a point-line geometry with nonempty point set $\mathcal{P}$, line set $\mathcal{L}$ and incidence relation $\mathrm{I} \subseteq \mathcal{P} \times \mathcal{L}$ such that any two distinct points are incident with at most one line. $\mathcal{S}$ is said to have $\operatorname{order}(s, t)$ if every line is incident with precisely $s+1$ points and every point is incident with exactly $t+1$ lines.

A partial linear space is called a generalized quadrangle [17] if every point is incident with at least two lines and if for every non-incident point-line pair $(x, L)$, there exists a unique point on $L$ collinear with $x$.

A partial linear space $\mathcal{S}$ is called a near hexagon if its collinearity graph has diameter 3 and if for every point $x$ and every line $L$, there exists a unique point on $L$ that is nearest to $x$ with respect to the distance in the collinearity graph. Such a near hexagon is said to be regular with parameters $\left(s, t, t_{2}\right)$ where $s, t, t_{2} \in \mathbb{N}$ if $\mathcal{S}$ has order $(s, t)$ and if every two points at distance 2 have precisely $t_{2}+1$ common neighbours. The regular near hexagons with parameters $\left(s, t, t_{2}\right)=(s, t, 0)$ are precisely the generalized hexagons of order $(s, t)$ (as for instance defined in [19]). If $x$ and $y$ are two points of $\mathcal{S}$, then $\mathrm{d}(x, y)$ denotes the distance between $x$ and $y$ (in the collinearity graph), and $\Gamma_{i}(x)$ with $i \in \mathbb{N}$ denotes the set of points at distance $i$ from $x$.

Suppose $\mathcal{S}$ is a near hexagon. A set $X$ of points of $\mathcal{S}$ is called a subspace if every line having two of its points in $X$ has all its points in $X$. If $X$ is a nonempty subspace, then the points of $X$ and the lines of $\mathcal{S}$ that have all their points in $X$ define a subgeometry 
$\widetilde{X}$ of $\mathcal{S}$. A set $X$ of points is called convex if every point on a shortest path between two points of $X$ is also contained in $X$. A quad of $\mathcal{S}$ is a nonempty convex subspace $Q$ for which $\widetilde{Q}$ is a generalized quadrangle.

Generalized quadrangles and near hexagons are special cases of so-called near polygons. These geometries were introduced in [18] because of their connection with line systems in Euclidean spaces. The family of near polygons includes the well-studied dual polar spaces and generalized polygons and also some "sporadic" examples related to finite simple groups. Some of these near polygons have recently been discovered $[2,3]$ and are related to the Suzuki chain, one of the most fundamental objects in the theory of finite simple groups. It also seems that near polygons form the natural setting for studying certain problems on (substructures of) dual polar spaces and generalized polygons, see e.g. [1].

Suppose now that $\mathcal{S}$ is a finite near hexagon with order $(s, t), s \geq 2$, having $v$ points. If $x$ and $y$ are two points at distance 2 from each other such that $\left|\Gamma_{1}(x) \cap \Gamma_{1}(y)\right| \geq 2$, then by Proposition 2.5 of [18], we know that $x$ and $y$ are contained in a unique quad $Q(x, y)$. If $Q$ is a quad of $\mathcal{S}$, then every line of $\widetilde{Q}$ is incident with precisely $s+1 \geq 3$ points, implying (see e.g. [5, Theorem 5.17]) that the generalized quadrangle $\widetilde{Q}$ must have some order. The following is the main result of this paper.

Theorem 1.1 Suppose $\mathcal{S}$ is a finite near hexagon with order $(s, t), s \geq 2$, having $v$ points. Let $L$ be a line of $\mathcal{S}$ and let $Q_{1}, Q_{2}, \ldots, Q_{k}$ with $k \in \mathbb{N}$ denote all quads through $L$. Suppose $Q_{i}$ with $i \in\{1,2, \ldots, k\}$ has order $\left(s, t_{2}^{(i)}\right)$. Then

$$
\sum_{i=1}^{k} \frac{\left(t_{2}^{(i)}\right)^{2}}{s+t_{2}^{(i)}} \geq t-\frac{s\left(s^{2}+1\right) v-s(s+1)\left(s^{2}+1\right)-s^{2} t(s+1)}{(s+1)\left(s^{4}-1\right)+s t(s-1)(s+1)^{2}+v} .
$$

In the special case where $\mathcal{S}$ is a regular near hexagon with parameters $\left(s, t, t_{2}\right)$ where $s \geq 2$ and $t_{2} \geq 1$, then $k=\frac{t}{t_{2}}, t_{2}^{(i)}=t_{2}$ for every $i \in\{1,2, \ldots, k\}$ and $v=(s+1)\left(1+s t+\frac{s^{2} t\left(t-t_{2}\right)}{t_{2}+1}\right)$. In this case, the bound mentioned in Theorem 1.1 reduces to $\left(t-t_{2}\right)\left(t-\left(s^{3}+t_{2}\left(s^{2}-s+\right.\right.\right.$ $1))) \leq 0$, i.e. to $t \leq s^{3}+t_{2}\left(s^{2}-s+1\right)$ since $t>t_{2}$. This bound is called the Mathon or Haemers-Mathon bound, see e.g. [9, 15]. Proofs of this bound can also be found in $[4,5,16]$.

In the special case where $\mathcal{S}$ is a generalized hexagon of order $(s, t), s \geq 2$, we have $k=0$ and $v=(s+1)\left(1+s t+s^{2} t^{2}\right)$ and the inequality mentioned in Theorem 1.1 reduces to $t \leq s^{3}$. This bound is known as the Haemers-Roos inequality, as it was discovered by Haemers and Roos in [10], see also Haemers [8].

In the special case that $\mathcal{S}$ is the direct product of a generalized quadrangle of order $\left(s, t_{2}\right), s, t_{2} \geq 2$, with a line of size $s+1$ ([4, (a)], [5, Section 6.6]) and $L$ is a line not contained in a quad of order $\left(s, t_{2}\right)$, then $t=t_{2}+1, v=(s+1)^{2}\left(s t_{2}+1\right), k=t_{2}+1$ and $t_{2}^{(i)}=1$ for every $i \in\{1,2, \ldots, k\}$. In this case, the inequality in Theorem 1.1 reduces to $t_{2} \leq s^{2}$. This is precisely the Higman inequality for generalized quadrangles $([11,12]$, $[17,1.2 .3])$. So, Theorem 1.1 can be regarded as a generalization of both the Higman 
inequality for generalized quadrangles and the Haemers-Roos inequality for generalized hexagons.

The known near hexagons for which equality can be obtained (in Theorem 1.1) are the generalized hexagons of order $\left(s, s^{3}\right)$, the direct products of generalized quadrangles of order $\left(s, s^{2}\right)$ with lines of size $s+1$, and the two sporadic near hexagons constructed in [18] from respectively the extended ternary Golay code and the Witt design $\mathrm{S}(5,8,24)$. It is not clear to the author whether other examples can exist or if there is extra information about the structure of the near hexagons that can be derived in case of equality.

For a given value of $s \in \mathbb{N} \backslash\{0,1\}$, there are only a limited number of possibilities for $(t, v)$ for which there can exist a near hexagon of order $(s, t)$ with $v$ points. This is a consequence of the fact that there exist upper bounds for $t$ and $v$ in terms of $s$ (like the bound $t \leq s^{4}+s^{2}$ from [7]) and strong divisibility conditions involving $s, t$ and $v$ (like the divisibility condition $\frac{s^{5} v}{(s+1)\left(s^{4}-1\right)+s t(s-1)(s+1)^{2}+v} \in \mathbb{N}$ from [6]). For given $s$, there are also a limited number of possibilities for the $t_{2}^{(i)}$ 's, as $\sqrt{s} \leq t_{2}^{(i)} \leq s^{2}$ if $t_{2}^{(i)} \neq 1$ and $\left(s+t_{2}^{(i)}\right) \mid s t_{2}^{(i)}(s+1)\left(t_{2}^{(i)}+1\right)([17$, Section 1.2]).

Not so many examples of (families of) finite near hexagons with an order $(s, t), s \geq 2$, are known, and recent research (as in [6]) has indicated that with respect to certain properties such near hexagons behave similarly as regular near hexagons (who have distanceregular collinearity graphs). The various recent restrictions (mentioned above) also indicate that for given $s$, there are relatively few surviving possibilities for $t$ and $v$. These facts have increased the interest in classifying near hexagons with an order (e.g. showing nonexistence). For certain of the feasible possibilities for $s, t$ and $v$, Theorem 1.1 offers information about the possible quads that can contain a given line, and this information can be useful during the classification process. For several of these possibilities, the right hand side of the inequality is strictly positive, implying that every line of the near hexagon is contained in a quad. The following consequence of Theorem 1.1 is interesting in view of the fact that there exist for every prime power $q$ a near hexagon of order $\left(q, q^{3}\right)$ without any quads (namely a dual twisted triality hexagon, see [19]).

Corollary 1.2 Let $\mathcal{S}$ be a finite near hexagon of order $(s, t)$ with $s \geq 2$ and $t>s^{3}$. Then every line of $\mathcal{S}$ is contained in at least one quad.

Proof. It suffices to show that in this case, the right hand side of the inequality is strictly positive, or equivalently

$$
s(s-1)(s+1)^{2} t^{2}+(s+1)\left(s^{4}+s^{2}-1\right) t+s(s+1)\left(s^{2}+1\right)-\left(s^{3}+s-t\right) v>0 .
$$

This is clearly the case if $t \geq s^{3}+s$. So, suppose $s^{3}+1 \leq t<s^{3}+s$.

Given a point $x$ of $\mathcal{S}$, we obviously have $\left|\Gamma_{0}(x)\right|=1,\left|\Gamma_{1}(x)\right|=s(t+1)$ and $\left|\Gamma_{2}(x)\right| \leq$ $\left|\Gamma_{1}(x)\right| \cdot s t=s^{2} t(t+1)$. As every point of $\Gamma_{2}(x)$ is collinear with at most st points of $\Gamma_{3}(x)$ and every point $y \in \Gamma_{3}(x)$ is collinear with exactly $t+1$ points of $\Gamma_{2}(x)$ (one on each line through $y$ ), we have $\left|\Gamma_{3}(x)\right| \leq \frac{1}{t+1} \cdot\left|\Gamma_{2}(x)\right| \cdot s t \leq s^{3} t^{2}$. Adding all contributions, we find that $v \leq 1+s(t+1)+s^{2} t(t+1)+s^{3} t^{2}=(s+1)\left(1+s t+s^{2} t^{2}\right)$. In combination 
with $0<s^{3}+s-t \leq s-1$, we see that the left hand side of (1) is bounded below by $s(s-1)(s+1)^{2} t^{2}+(s+1)\left(s^{4}+s^{2}-1\right) t+s(s+1)\left(s^{2}+1\right)-\left(s^{2}-1\right)\left(1+s t+s^{2} t^{2}\right)>0$.

The proof of Theorem 1.1 uses similar ideas as the proofs of the Haemers-Roos inequality in $[8,10]$ and the Haemers-Mathon bound in [9]. As in these papers, we consider two matrices $C$ and $\widetilde{C}$ such that $\widetilde{C}$ is the principle submatrix of $C$ defined by the points at distance 1 from a given line $L$. The trivial inequality $\operatorname{rank}(\widetilde{C}) \leq \operatorname{rank}(C)$ then leads to the inequality mentioned in Theorem 1.1. Although the methods are similar to those in $[8,9,10]$, there are also important differences.

The computation of $\operatorname{rank}(C)$ in the case of generalized hexagons and regular near hexagons was realized in $[8,9,10]$ using the 3 -class association scheme on the point set. Such an association scheme does not necessarily exist for general finite near hexagons with an order. Despite this fact, we still are able to compute the rank of $C$ by relying on some recent results of [7].

In the case of generalized hexagons, the matrix $\widetilde{C}$ has an easy form and its rank is readily computed, see $[8,10]$. In general, $\widetilde{C}$ can be written as the sum of two matrices, where one of them is a "block diagonal matrix". In the case of regular near hexagons, these two matrices commute and have a common basis of eigenvectors. This fact was essentially used in [9] to compute the eigenvalues with their multiplicities and (subsequently) the rank of the matrix $\widetilde{C}$. In the case of general finite near hexagons with an order, these two matrices do not necessarily commute. We shall therefore use another method to compute $\operatorname{rank}(\widetilde{C})$.

The computation of $\operatorname{rank}(C)$ will be done in Section 2. The computation of $\operatorname{rank}(\widetilde{C})$ will be realized in Section 5 by relying on some helpful results from Sections 3 and 4 . Throughout the paper, we follow the following notation. If $m \in \mathbb{N} \backslash\{0\}$, then $O_{m}$ denotes the $m \times m$ zero-matrix, $I_{m}$ denotes the $m \times m$ identity matrix and $J_{m}$ the matrix all whose entries are equal to 1 (with all matrices being defined over the reals).

\section{Some properties of finite near hexagons with an order}

In this section, $\mathcal{S}=(\mathcal{P}, \mathcal{L}, \mathrm{I})$ denotes a finite near hexagon of order $(s, t), s \geq 2$, having $v=|\mathcal{P}|$ points. We define the following numbers:

$$
n_{0}:=1, n_{1}:=s(t+1), n_{2}:=\frac{v}{s+1}-1+s^{2} t-s t, n_{3}:=\frac{s v}{s+1}-s^{2} t-s .
$$

For a proof of the following lemma, see e.g. Proposition 2.3 of [7].

Lemma 2.1 For every point $x$ and every $i \in\{0,1,2,3\}$, we have $\left|\Gamma_{i}(x)\right|=n_{i}$. 
We also define the following number:

$$
\alpha:=n_{0}+\frac{n_{1}}{s^{2}}+\frac{n_{2}}{s^{4}}+\frac{n_{3}}{s^{6}}=\frac{(s+1)\left(s^{4}-1\right)+s t(s-1)(s+1)^{2}+v}{s^{5}} .
$$

We then have $\alpha>n_{0}+\frac{n_{1}}{s^{2}}=1+\frac{t+1}{s}$.

Let $p_{1}, p_{2}, \ldots, p_{v}$ be a particular ordering of the points of $\mathcal{S}$. For every $i \in\{0,1,2,3\}$, let $A_{i}$ be the symmetric real $v \times v$ matrix whose $(j, k)$ th entry with $1 \leq j, k \leq v$ is defined as

$$
\left(A_{i}\right)_{j, k}:= \begin{cases}1 & \text { if }\left(p_{j}, p_{k}\right)=i \\ 0 & \text { otherwise }\end{cases}
$$

Then $A_{0}$ is the $v \times v$ identity matrix $I_{v}, A$ is the collinearity matrix of $\mathcal{S}$ and $A_{0}+A_{1}+$ $A_{2}+A_{3}=J_{v}$. We define the following additional symmetric matrix $N$ :

$$
N:=A_{0}-\frac{A_{1}}{s}+\frac{A_{2}}{s^{2}}-\frac{A_{3}}{s^{3}} .
$$

In the sequel, we put $O:=O_{v}, I:=I_{v}$ and $J:=J_{v}$.

Lemma 2.2 We have $J^{2}=v \cdot J, N J=J N=O$ and $N^{2}=\alpha \cdot N$.

Proof. Obviously, we have $J^{2}=v \cdot J$. By Proposition 4.1 of [7], we know that $N^{2}=\alpha \cdot N$. By Lemma 2.1 and the definition of the matrix $N$, we know that $J \cdot N=N \cdot J=\alpha^{\prime} \cdot J$, where $\alpha^{\prime}=n_{0}-\frac{n_{1}}{s}+\frac{n_{2}}{s^{2}}-\frac{n_{3}}{s^{3}}=0$.

Lemma 2.3 - If $v=s^{3} \alpha$, then the eigenvalues of the matrix $s^{3} N+J$ are 0 and $v$ and their respective multiplicities are equal to $v-\left(s^{3}+1\right)=s^{3}(\alpha-1)-1>0$ and $s^{3}+1$.

- If $v \neq s^{3} \alpha$, then the eigenvalues of the matrix $s^{3} N+J$ are equal to $0, s^{3} \alpha$ and $v$, and their respective multiplicities are equal to $v-1-\frac{v}{\alpha}>0, \frac{v}{\alpha}$ and 1 .

Proof. Since the matrices $I=A_{0}, J$ and $N$ are linearly independent, the minimal polynomial of $s^{3} N+J$ has degree at least 2. Based on Lemma 2.2, we compute that

$$
\begin{aligned}
s^{3} N+J & =s^{3} N+J \\
\left(s^{3} N+J\right)^{2} & =s^{6} \alpha N+v J \\
\left(s^{3} N+J\right)^{3} & =\left(s^{6} \alpha N+v J\right) \cdot\left(s^{3} N+J\right)=s^{9} \alpha^{2} N+v^{2} J
\end{aligned}
$$

So, we see that the minimal polynomial of $s^{3} N+J$ has degree 2 if and only if $v=s^{3} \alpha$, in which case $X^{2}-v X$ is the minimal polynomial with roots 0 and $v$.

If $v \neq s^{3} \alpha$, then the minimal polynomial is equal to

$$
\left|\begin{array}{ccc}
X & s^{3} & 1 \\
X^{2} & s^{6} \alpha & v \\
X^{3} & s^{9} \alpha^{2} & v^{2}
\end{array}\right|=s^{3}\left(v-s^{3} \alpha\right) \cdot X\left(X-s^{3} \alpha\right)(X-v),
$$


which has $0, s^{3} \alpha$ and $v$ as roots.

Suppose $v=s^{3} \alpha$. Let $m_{1}$ and $m_{2}$ denote the respective multiplicities of the eigenvalues 0 and $v$. Then $m_{1}+m_{2}=v$ and $0 \cdot m_{1}+v \cdot m_{2}=\operatorname{Tr}\left(s^{3} N+J\right)=\left(s^{3}+1\right) v$, implying that $m_{2}=s^{3}+1$ and $m_{1}=v-\left(s^{3}+1\right)=(\alpha-1) s^{3}-1$. As $\alpha-1>\frac{t+1}{s}$, we have $m_{1}>0$.

Suppose $v \neq s^{3} \alpha$. Then let $m_{1}, m_{2}$ and $m_{3}$ denote the respective multiplicities of $0, v$ and $s^{3} \alpha$. Then

$$
\begin{aligned}
m_{1}+m_{2}+m_{3} & =v \\
v \cdot m_{2}+s^{3} \alpha \cdot m_{3} & =\operatorname{Tr}\left(s^{3} N+J\right)=v\left(s^{3}+1\right), \\
v^{2} \cdot m_{2}+s^{6} \alpha^{2} \cdot m_{3} & =\operatorname{Tr}\left(\left(s^{3} N+J\right)^{2}\right)=\operatorname{Tr}\left(s^{6} \alpha N+v J\right)=v\left(s^{6} \alpha+v\right) .
\end{aligned}
$$

This implies that

$$
m_{1}=v-1-\frac{v}{\alpha}, \quad m_{2}=1, \quad m_{3}=\frac{v}{\alpha} .
$$

Suppose $m_{1}=0$. Then $\alpha=\frac{v}{v-1}>1+\frac{t+1}{s}$, i.e. $s>(t+1)(v-1)$, in contradiction with $v \geq s+1$. Hence, $m_{1}>0$.

Lemma 2.4 The matrix $C:=A_{2}-(s-1) A_{1}+\left(s^{2}-s+1\right) A_{0}$ has rank $\frac{v}{\alpha}+1$.

Proof. An easy computation shows that

$$
\frac{s^{3} N+J}{s+1}=A_{2}-(s-1) A_{1}+\left(s^{2}-s+1\right) A_{0},
$$

and by Lemma 2.3 we know that the matrix $s^{3} N+J$ has rank $\frac{v}{\alpha}+1$ (regardless of whether $v=s^{3} \alpha$ or not).

\section{Some ranks of matrices associated with generalized quadrangles of order $\left(s, t_{2}\right)$}

Let $\mathcal{Q}$ be a finite generalized quadrangle of order $\left(s, t_{2}\right)$ with $s, t_{2} \geq 1$, and let $L$ be a line of $\mathcal{Q}$. We denote by $X$ the set of all points of $\mathcal{Q}$ not contained in $L$. For every $x \in X$, let $x^{\prime}$ denote the unique point on $L$ collinear with $x$. We consider the following symmetric relations on the set $X$ :

- $R_{0}=\{(x, x) \mid x \in X\}$;

- $R_{1}=\left\{(x, y) \in X \times X \mid \mathrm{d}(x, y)=1\right.$ and $\left.x^{\prime}=y^{\prime}\right\}$;

- $R_{2}=\left\{(x, y) \in X \times X \mid \mathrm{d}(x, y)=1\right.$ and $\left.x^{\prime} \neq y^{\prime}\right\}$;

- $R_{3}=\left\{(x, y) \in X \times X \mid \mathrm{d}(x, y)=2\right.$ and $\left.x^{\prime}=y^{\prime}\right\}$;

- $R_{4}=\left\{(x, y) \in X \times X \mid \mathrm{d}(x, y)=2\right.$ and $\left.x^{\prime} \neq y^{\prime}\right\}$. 
Put $v:=|X|=(s+1) s t_{2}, O:=O_{v}, I:=I_{v}$ and $J:=J_{v}$. Let $p_{1}, p_{2}, \ldots, p_{v}$ be a particular ordering of the elements of $X$. With the relation $R_{k}, k \in\{0,1,2,3,4\}$, we associate a real symmetric matrix $B_{k}=\left(b_{i j}^{k}\right)_{1 \leq i, j \leq v}$, whose entries are defined as follows:

$$
b_{i j}^{(k)}:= \begin{cases}1 & \text { if }\left(p_{i}, p_{j}\right) \in R_{k} \\ 0 & \text { otherwise }\end{cases}
$$

Then $B_{0}=I$ and $B_{0}+B_{1}+B_{2}+B_{3}+B_{4}=J$. Moreover, we have

$$
B_{0} J=J, B_{1} J=(s-1) J, B_{2} J=s t_{2} J, B_{3} J=s\left(t_{2}-1\right) J, B_{4} J=s(s-1) t_{2} J .
$$

We also have $B_{1}=O$ if and only if $s=1, B_{2} \neq O, B_{3}=O$ if and only if $t_{2}=1$ and $B_{4}=O$ if and only if $s=1$. We define the following real $5 \times 5$ matrix:

$$
M=\left[\begin{array}{ccccc}
s^{2}-s+1 & -(s-1) & -(s-1) & 1 & 1 \\
-(s-1)^{2} & 2 s-1 & s-1 & s-1 & -1 \\
-s(s-1) t_{2} & s t_{2} & s t_{2} & 0 & 0 \\
s\left(t_{2}-1\right) & s\left(t_{2}-1\right) & 0 & s\left(t_{2}-1\right) & 0 \\
s(s-1) t_{2} & -s t_{2} & 0 & 0 & s t_{2}
\end{array}\right]
$$

The following can easily be verified (e.g. with Maple).

Lemma 3.1 - The eigenvalues of $M$ are equal to $s^{2}+s t_{2}$ (multiplicity 1$)$, st $t_{2}$ (multiplicity 2) and 0 (multiplicity 2).

- The minimal polynomial of $M$ is equal to $m(X):=X\left(X-s t_{2}\right)\left(X-\left(s^{2}+s t_{2}\right)\right) \in$ $\mathbb{R}[X]$.

Lemma 3.2 The following equations hold:

$$
\begin{aligned}
& B_{1} B_{0}=B_{1} \\
& B_{1} B_{1}=(s-1) B_{0}+(s-2) B_{1} \\
& B_{1} B_{2}=B_{4} \\
& B_{1} B_{3}=(s-1) B_{3}, \\
& B_{1} B_{4}=(s-1) B_{2}+(s-2) B_{4}, \\
& B_{2} B_{0}=B_{2} \\
& B_{2} B_{1}=B_{4} \\
& B_{2} B_{2}=s t_{2} B_{0}+(s-1) B_{2}+t_{2} B_{3}+\left(t_{2}-1\right) B_{4}, \\
& B_{2} B_{3}=\left(t_{2}-1\right) B_{2}+\left(t_{2}-1\right) B_{4} \\
& B_{2} B_{4}=s t_{2} B_{1}+(s-1)\left(t_{2}-1\right) B_{2}+(s-1) t_{2} B_{3}+\left(s t_{2}-2 t_{2}+1\right) B_{4} .
\end{aligned}
$$

Proof. Obviously, $B_{i} B_{0}=B_{i} I=B_{i}$ for every $i \in\{1,2\}$. 
If $(x, y) \in R_{1}$ and $(y, z) \in R_{1}$, then $(x, z)$ belongs to either $R_{0}$ and $R_{1}$. If $(x, z) \in R_{0}$, i.e. if $x=z$, then there are $s-1$ points $y \in X$ such that $(x, y),(y, z) \in R_{1}$, namely the $s-1$ points on the line $x x^{\prime}$ distinct from $x$ and $x^{\prime}$. If $(x, z) \in R_{1}$, then there are $s-2$ points $y \in X$ such that $(x, y),(y, z) \in R_{1}$, namely the $s-2$ points on $x x^{\prime}$ distinct from $x$, $z$ and $x^{\prime}$. We conclude that $B_{1} B_{1}=(s-1) B_{0}+(s-2) B_{1}$.

If $(x, y) \in R_{1}$ and $(y, z) \in R_{2}$, then the lines $x y$ and $y z$ are different, and so we necessarily have $\mathrm{d}(x, z)=2$. Since $x^{\prime}=y^{\prime}$ and $y^{\prime} \neq z^{\prime}$, we have $x^{\prime} \neq z^{\prime}$, implying that $(x, z) \in R_{4}$. Conversely, if $(x, z) \in R_{4}$, then there is a unique point $y \in X$ for which $(x, y) \in R_{1}$ and $(y, z) \in R_{2}$, namely the unique point on $x x^{\prime}$ collinear with $z$. We conclude that $B_{1} B_{2}=B_{4}$. Taking the transpose of this equation, we find $B_{2} B_{1}=B_{4}$.

If $(x, y) \in R_{1}$ and $(y, z) \in R_{3}$, then also $(x, z) \in R_{3}$. Conversely, if $(x, z) \in R_{3}$, then there are $s-1$ points $y \in X$ such that $(x, y) \in R_{1}$ and $(y, z) \in R_{3}$, namely the $s-1$ points on $x x^{\prime}$ distinct from $x$ and $x^{\prime}$. We conclude that $B_{1} B_{3}=(s-1) B_{3}$.

We compute that $B_{1} B_{4}=B_{1} J-B_{1} B_{0}-B_{1} B_{1}-B_{1} B_{2}-B_{1} B_{3}=(s-1) B_{2}+(s-2) B_{4}$.

Suppose $(x, y)$ and $(y, z)$ belong to $R_{2}$. It is impossible that $(x, z) \in R_{1}$, as otherwise $x, y, z$ are mutually collinear and so contained in a line that would be disjoint from $L$ (since $\left.(x, y) \in R_{2}\right)$ and also meet $L$ (since $(x, z) \in R_{1}$ ). So, $(x, z) \in R_{0} \cup R_{2} \cup R_{3} \cup R_{4}$.

If $(x, z) \in R_{0}$, i.e. $x=z$, then there are st $t_{2}$ points $y$ such that $(x, y),(y, z) \in R_{2}$, namely the $s t_{2}$ points $y \in X \backslash\{x\}$ that are on one of the $t_{2}$ lines through $x$ disjoint from $L$. If $(x, z) \in R_{2}$, then there are $s-1$ points $y \in X$ such that $(x, y),(y, z) \in R_{2}$, namely the $s-1$ points on the line $x z$ distinct from $x$ and $z$. If $(x, z) \in R_{3}$, then there are $t_{2}$ points $y \in X$ for which $(x, y),(y, z) \in R_{2}$, namely the $t_{2}$ neighbours of $x$ and $z$ distinct from $x^{\prime}=z^{\prime}$. If $(x, z) \in R_{4}$, then there are $t_{2}-1$ points $y \in X$ for which $(x, y),(y, z) \in R_{2}$, namely the $t_{2}-1$ neighbours of $x$ and $z$ that do not lie on the lines $x x^{\prime}$ and $z z^{\prime}$.

We conclude that $B_{2} B_{2}=s t_{2} B_{0}+(s-1) B_{2}+t_{2} B_{3}+\left(t_{2}-1\right) B_{4}$.

Suppose $(x, y) \in R_{2}$ and $(y, z) \in R_{3}$. Then $x^{\prime} \neq y^{\prime}$ and $y^{\prime}=z^{\prime}$ implies that $x^{\prime} \neq z^{\prime}$, i.e. $(x, z) \in R_{2} \cup R_{4}$.

Suppose $(x, z) \in R_{2}$. Any point $y \in X$ satisfying $(x, y) \in R_{2}$ and $(y, z) \in R_{3}$ cannot be contained in any of the lines $x x^{\prime}, x z$ and so must be contained in one of the $t_{2}-1$ lines through $x$ distinct from $x x^{\prime}$ and $x z$. Each of these $t_{2}-1$ lines contains a unique point $y \in X$ satisfying $(x, y) \in R_{2}$ and $(y, z) \in R_{3}$, namely the unique point of that line collinear with $z^{\prime}$.

Suppose $(x, z) \in R_{4}$. Any point $y \in X$ satisfying $(x, y) \in R_{2}$ and $(y, z) \in R_{3}$ cannot be contained on $x x^{\prime}$ nor on the unique line through $x$ meeting $z z^{\prime}$. On each of the $t_{2}-1$ other lines through $x$, there is a unique choice for such a point, namely the unique point on that line collinear with $z^{\prime}$.

It follows that $B_{2} B_{3}=\left(t_{2}-1\right) B_{2}+\left(t_{2}-1\right) B_{4}$.

We compute that $B_{2} B_{4}=B_{2} J-B_{2} B_{0}-B_{2} B_{1}-B_{2} B_{2}-B_{2} B_{3}=s t_{2} B_{1}+(s-1)\left(t_{2}-\right.$ 1) $B_{2}+(s-1) t_{2} B_{3}+\left(s t_{2}-2 t_{2}+1\right) B_{4}$.

The following is an immediate consequence of Lemma 3.2. 
Corollary 3.3 Putting $F:=J-s\left(B_{1}+B_{2}\right)+\left(s^{2}-s\right) I$, we find

$$
\begin{aligned}
& F B_{0}=\left(s^{2}-s+1\right) B_{0}-(s-1) B_{1}-(s-1) B_{2}+B_{3}+B_{4}, \\
& F B_{1}=-(s-1)^{2} B_{0}+(2 s-1) B_{1}+(s-1) B_{2}+(s-1) B_{3}-B_{4}, \\
& F B_{2}=-s(s-1) t_{2} B_{0}+s t_{2} B_{1}+s t_{2} B_{2}, \\
& F B_{3}=s\left(t_{2}-1\right) B_{0}+s\left(t_{2}-1\right) B_{1}+s\left(t_{2}-1\right) B_{3}, \\
& F B_{4}=s(s-1) t_{2} B_{0}-s t_{2} B_{1}+s t_{2} B_{4} .
\end{aligned}
$$

The equations in Corollary 3.3 can be summarized as

$$
\left[\begin{array}{lllll}
B_{0} & B_{1} & B_{2} & B_{3} & B_{4}
\end{array}\right]^{T} \cdot F=(M \otimes I) \cdot\left[\begin{array}{lllll}
B_{0} & B_{1} & B_{2} & B_{3} & B_{4}
\end{array}\right]^{T},
$$

where $M \otimes I$ denotes the Kronecker product [13, Section 4.2] of the matrices $M$ and $I$. If $p(X) \in \mathbb{R}[X]$, then by Lemma 4.2 .10 of [13], we know that

$$
\left[\begin{array}{lllll}
B_{0} & B_{1} & B_{2} & B_{3} & B_{4}
\end{array}\right]^{T} \cdot p(F)=(p(M) \otimes I) \cdot\left[\begin{array}{lllll}
B_{0} & B_{1} & B_{2} & B_{3} & B_{4}
\end{array}\right]^{T} .
$$

In particular, we have $\left[\begin{array}{lllll}B_{0} & B_{1} & B_{2} & B_{3} & B_{4}\end{array}\right]^{T} \cdot m(F)=O$, where $m(X) \in \mathbb{R}[X]$ is the polynomial as defined in Lemma 3.1. This implies that $m(F)=B_{0} m(F)=O$. So, the minimal polynomial of $F$ is a divisor of $m(X)$ and its eigenvalues are $\lambda_{1}:=s^{2}+s t_{2}$, $\lambda_{2}:=s t_{2}$ and $\lambda_{3}:=0$ (possibly with multiplicity 0 ). For every $i \in\{1,2,3\}$, let $m_{i}$ denote the multiplicity of $\lambda_{i}$ as an eigenvalue of $F$. Then

$$
\begin{cases}m_{1}+m_{2}+m_{3} & =v=s t_{2}(s+1), \\ m_{1} \lambda_{1}+m_{2} \lambda_{2}+m_{3} \lambda_{3} & =\operatorname{Tr}(F), \\ m_{1} \lambda_{1}^{2}+m_{2} \lambda_{2}^{2}+m_{3} \lambda_{3}^{2} & =\operatorname{Tr}\left(F^{2}\right) .\end{cases}
$$

This linear system allows to compute the values for $m_{1}, m_{2}$ and $m_{3}$ as soon as we know the values of $\operatorname{Tr}(F)$ and $\operatorname{Tr}\left(F^{2}\right)$. Since $F=J-s\left(B_{1}+B_{2}\right)+\left(s^{2}-s\right) I$, we have $\operatorname{Tr}(F)=\left(s^{2}-s+1\right) s t_{2}(s+1)$. We compute

$$
\begin{aligned}
F^{2}= & J^{2}+s^{2}\left(B_{1}^{2}+B_{2}^{2}+2 B_{1} B_{2}\right)+\left(s^{2}-s\right)^{2} I \\
& -2 s\left(B_{1}+B_{2}\right) J+2\left(s^{2}-s\right) J-2 s^{2}(s-1)\left(B_{1}+B_{2}\right) \\
= & s t_{2}(s+1) J+s^{2}\left((s-1) I+(s-2) B_{1}+s t_{2} I+(s-1) B_{2}+t_{2} B_{3}+\left(t_{2}-1\right) B_{4}\right. \\
& \left.+2 B_{4}\right)+\left(s^{2}-s\right)^{2} I-2 s\left(s-1+s t_{2}\right) J+2\left(s^{2}-s\right) J-2 s^{2}(s-1)\left(B_{1}+B_{2}\right) .
\end{aligned}
$$

From this one easily deduces that $\operatorname{Tr}\left(F^{2}\right)=s^{2} t_{2}(s+1)\left(s^{2} t_{2}-s t_{2}+s^{3}-s^{2}+t_{2}\right)$. The above linear system can now be solved and we find the following solutions:

$$
m_{1}=\frac{\left(s^{2}-1\right) s t_{2}}{s+t_{2}}, \quad m_{2}=s+1, \quad m_{3}=\frac{s+1}{s+t_{2}}\left(s t_{2}^{2}+s t_{2}-s-t_{2}\right) .
$$

From this, one easily deduces that $\operatorname{rank}(F)=s+1+\frac{\left(s^{2}-1\right) s t_{2}}{s+t_{2}}$. 
We thus see that the eigenspace corresponding to the eigenvalue $s t_{2}$ has degree $s+1$. In fact, it is easy to describe this eigenspace. Put $L=\left\{x_{1}, x_{2}, \ldots, x_{s+1}\right\}$. We may suppose that we have ordered the points $p_{1}, p_{2}, \ldots, p_{v}$ in such a way that the points in $\Gamma_{1}\left(x_{1}\right)$ come first, followed by those in $\Gamma_{1}\left(x_{2}\right)$, then those in $\Gamma_{1}\left(x_{3}\right)$, etc. For every $i \in\{1,2, \ldots, s+1\}$, let $w_{i}$ be the column vector of dimensions $s t_{2}(s+1) \times 1$ such that if $j \in\left\{1,2, \ldots, s t_{2}(s+1)\right\}$, then $\left(w_{i}\right)_{j 1}=1$ if $(i-1) s t_{2}+1 \leq j \leq i \cdot s t_{2}$ and $\left(w_{i}\right)_{j 1}=0$ otherwise. Then $\left\langle w_{1}, w_{2}, \ldots, w_{s+1}\right\rangle$ is the $(s+1)$-dimensional eigenspace of $F$ corresponding to the eigenvalue $s t_{2}$.

Now, define $E:=B_{0}+B_{1}+B_{3}$. Taking the ordering of the points as above, we see that $E$ is a block diagonal matrix whose diagonal consists of $s+1$ blocks of type $J_{s t_{2}}$. So, $E$ has two eigenvalues, namely $s t_{2}$ with multiplicity $s+1$ and 0 with multiplicity $\left(s t_{2}-1\right)(s+1)$. The eigenspace corresponding to the eigenvalue $s t_{2}$ is obviously equal to $\left\langle w_{1}, w_{2}, \ldots, w_{s+1}\right\rangle$.

So, if $W_{i}$ with $i \in\{1,2,3\}$ denotes the eigenspace of $F$ corresponding to the eigenvalue $\lambda_{i}$, then $W_{2}=\left\langle w_{1}, w_{2}, \ldots, w_{s+1}\right\rangle$ and $\mathbb{R}^{n}$ is the orthogonal direct sum $W_{1} \oplus W_{2} \oplus W_{3}$. As $W_{1} \oplus W_{3}$ is orthogonal with $W_{2}$, it should be the eigenspace of $E$ corresponding to the eigenvalue 0 .

Lemma 3.4 If $E=B_{0}+B_{1}+B_{3}$ and $F=J-s\left(B_{1}+B_{2}\right)+\left(s^{2}-s\right) I=\left(B_{3}+B_{4}\right)-$ $(s-1)\left(B_{1}+B_{2}\right)+\left(s^{2}-s+1\right) I$, then the following hold:

(1) The matrix E has rank $s+1$.

(2) The matrix $F-E$ has rank $\frac{\left(s^{2}-1\right) s t_{2}}{s+t_{2}}$.

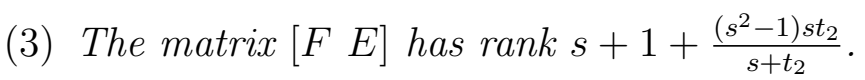

Proof. (1) The matrix $E$ has only one nonzero eigenvalue, namely $s t_{2}$, whose corresponding eigenspace $W_{2}$ has dimension $s+1$.

(2) The matrix $F-E$ has two eigenvalues, namely $s^{2}+s t_{2}=s^{2}+s t_{2}-0$ and $0=s t_{2}-s t_{2}=0-0$, whose respective eigenspaces are $W_{1}$ and $\left\langle W_{2}, W_{3}\right\rangle$. So, the rank of $F-E$ is equal to $\operatorname{dim}\left(W_{1}\right)=m_{1}=\frac{\left(s^{2}-1\right) s t_{2}}{s+t_{2}}$.

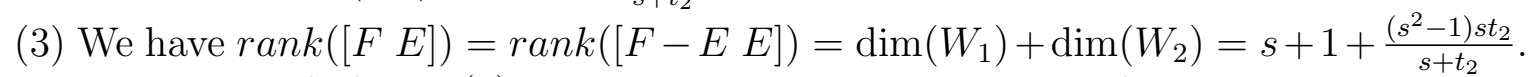
Indeed, by the proof of part (2) we know that the column space of $F-E$ is equal to $W_{1}$, and by the proof of part (1) we know that the column space of $E$ is equal to $W_{2}$.

Remark. The eigenvalues and multiplicities of the matrix $B_{1}+B_{2}$ were mentioned in [9]. From this information, Lemma 3.4 can also be derived. We have opted to give a self-contained proof of Lemma 3.4 since [9] was never published and only mentions the values of eigenvalues and multiplicities.

\section{Some ranks of other matrices}

For given $s, t \in \mathbb{N} \backslash\{0\}$, consider the partial linear space $\mathcal{S}$ that satisfies the following properties: 
(1) Every line contains precisely $s+1$ points.

(2) There exists a line $L$ in $\mathcal{S}$ that meets every other line.

(3) Every point of $L$ is incident with precisely $t$ lines distinct from $L$.

(4) Every point not incident with $L$ is incident with a unique line meeting $L$.

Alternatively, $\mathcal{S}$ can also be obtained by considering a line pencil $\mathcal{U}$ consisting of $t$ lines of size $s+1$ through a given point (the center), taking $s+1$ isomorphic copies of $\mathcal{U}$ and adding one extra line that contains the $s+1$ centers of these $s+1$ isomorphic line pencils.

Let $X$ denote the set of all points of $\mathcal{S}$ not contained in $L$. Similarly as in Section 3 , we can define relations $R_{0}, R_{1}, R_{2}, R_{3}$ and $R_{4}$ on $X$. As every line meets $L$, we have $R_{2}=\emptyset$. If $x$ and $y$ are two noncollinear points of $X$, then any path between $x$ and $y$ must pass through their projections $x^{\prime}$ and $y^{\prime}$ on the line $L$, showing that also $R_{4}=\emptyset$. Note that if $t=1$, then also $R_{3}=\emptyset$.

If $A$ is a square matrix and $k \in \mathbb{N} \backslash\{0\}$, then $\mathcal{D}(A, k)$ denotes the block diagonal matrix whose diagonal consists of $k$ copies of the matrix $A$.

Put $v:=|X|=s t(s+1)$. Similarly as in Section 3, we can associate to each $R_{i}$, $i \in\{0,1,2,3,4\}$, a real $v \times v$ matrix $B_{i}$. We have $B_{0}=I_{v}$ and $B_{2}=B_{4}=O_{v}$.

Lemma 4.1 If $E:=B_{0}+B_{1}+B_{3}$ and $F:=\left(B_{3}+B_{4}\right)-(s-1) \cdot\left(B_{1}+B_{2}\right)+\left(s^{2}-s+1\right) I_{v}=$ $B_{3}-(s-1) B_{1}+\left(s^{2}-s+1\right) I_{v}$, then the following hold:

(1) $\operatorname{rank}(E)=s+1$;

(2) $\operatorname{rank}(F-E)=t\left(s^{2}-1\right)$;

(3) $\operatorname{rank}\left(\left[\begin{array}{ll}F & E\end{array}\right]\right)=s+1+t\left(s^{2}-1\right)$.

Proof. Put $L=\left\{x_{1}, x_{2}, \ldots, x_{s+1}\right\}$. Without loss of generality, we may assume that we have labeled the points in $X$ in such a way that those collinear with $x_{1}$ come first, followed by those collinear with $x_{2}$, etc. The collinearity graph $\Gamma$ of $\mathcal{S}$ is the disjoint union of $(s+1) t$ cliques of size $s$, and we may suppose that we have labeled the points in each $\Gamma_{1}\left(x_{i}\right)$ in such a way that those belonging to the same clique occupy consecutive places. Then

$$
\begin{aligned}
B_{0} & =I_{v}, \\
B_{0}+B_{1} & =U_{1}:=\mathcal{D}\left(J_{s},(s+1) t\right), \\
B_{0}+B_{1}+B_{3} & =U_{2}:=\mathcal{D}\left(J_{s t}, s+1\right) .
\end{aligned}
$$

We thus see that $E=U_{2}$ has rank $s+1$. We compute that

$$
\begin{aligned}
F & =B_{3}-(s-1) B_{1}+\left(s^{2}-s+1\right) I_{v} \\
& =\left(U_{2}-U_{1}\right)-(s-1)\left(U_{1}-I_{v}\right)+\left(s^{2}-s+1\right) I_{v} \\
& =U_{2}-s U_{1}+s^{2} I_{v} .
\end{aligned}
$$




\begin{tabular}{|c||c|c|c|c|c|c|}
\hline Eigenspace & Dimension & $\widetilde{E}=\widetilde{U_{2}}$ & $\widetilde{U_{1}}$ & $\widetilde{I_{v}}$ & $\widetilde{F}:=\widetilde{U_{2}}-s \widetilde{U}_{1}+s^{2} \widetilde{I}_{v}$ & $\widetilde{F}-\widetilde{E}=-s \widetilde{U}_{1}+s^{2} \widetilde{I}_{v}$ \\
\hline \hline$\langle\bar{w}\rangle$ & 1 & $s t$ & $s$ & 1 & $s t$ & 0 \\
\hline$W_{1}$ & $t-1$ & 0 & $s$ & 1 & 0 & 0 \\
\hline$W_{2}$ & $(s-1) t$ & 0 & 0 & 1 & $s^{2}$ & $s^{2}$ \\
\hline
\end{tabular}

Table 1: Eigenvalues of the matrices $\widetilde{E}=\widetilde{U_{2}}, \widetilde{U_{1}}, \widetilde{I_{v}}, \widetilde{F}$ and $\widetilde{F}-\widetilde{E}$

If we put $\widetilde{U_{2}}=J_{s t}, \widetilde{U_{1}}:=\mathcal{D}\left(J_{s}, t\right), \widetilde{I_{v}}:=I_{s t}, \widetilde{E}:=\widetilde{U_{2}}$ and $\widetilde{F}:=\widetilde{U_{2}}-s \widetilde{U_{1}}+s^{2} \widetilde{I}_{v}$, then $F=\mathcal{D}(\widetilde{F}, s+1)$ and $E=\mathcal{D}(\widetilde{E}, s+1)$.

Consider the following column vectors of dimensions $s t \times 1$ :

$$
\begin{aligned}
& \bar{w}=\left[\begin{array}{lllll}
1 & 1 & \cdots & 1 & 1
\end{array}\right]^{T},
\end{aligned}
$$

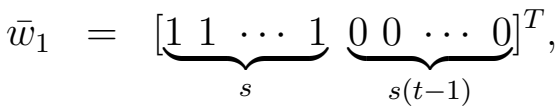

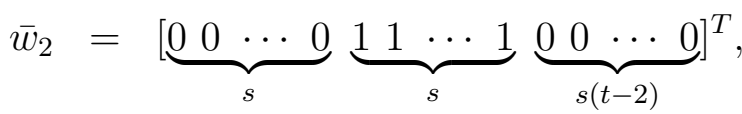

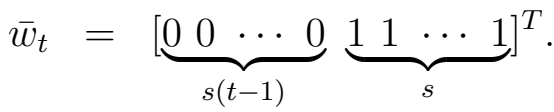

Put $W_{1}=\left\langle\bar{w}_{1}-\bar{w}_{2}, \ldots, \bar{w}_{1}-\bar{w}_{t}\right\rangle$ and let $W_{2}$ denote the subspace $\left\langle\bar{w}_{1}, \bar{w}_{2}, \ldots, \bar{w}_{t}\right\rangle^{\perp}$ consisting of all column vectors of dimensions st $\times 1$ that are orthogonal with each of $\bar{w}_{1}, \bar{w}_{2}, \ldots, \bar{w}_{t}$ with respect to the standard inner product. Then $\mathbb{R}^{s t}$ is the direct sum $\langle\bar{w}\rangle \oplus W_{1} \oplus W_{2}$.

The matrices $\widetilde{U_{2}}, \widetilde{U_{1}}$ and $\widetilde{I_{v}}$ commute and so they have a common basis of eigenvectors, see e.g. [14, Theorem 1.3.21]. Such a basis can be taken to be equal to $\{\bar{w}\} \cup B_{1} \cup B_{2}$, where $B_{i}$ with $i \in\{1,2\}$ is a basis of $W_{i}$. The corresponding eigenvalues are mentioned in Table 1. From this table, we deduce that

- $\operatorname{rank}(\widetilde{E})=1$ and $\operatorname{rank}(\widetilde{F}-\widetilde{E})=(s-1) t$,

- the column space of $\widetilde{F}-\widetilde{E}$ is $W_{2}$ and the one of $\widetilde{E}$ is $\langle\bar{w}\rangle$,

- $\operatorname{rank}([\widetilde{F} \widetilde{E}])=\operatorname{rank}([\widetilde{F}-\widetilde{E} \widetilde{E}])=\operatorname{dim}\left(\left\langle W_{2}, \bar{w}\right\rangle\right)=1+(s-1) t$.

Since $F=\mathcal{D}(\widetilde{F}, s+1)$ and $E=\mathcal{D}(\widetilde{E}, s+1)$, the claims of the lemma follow.

\section{Proof of Theorem 1.1}

Let $\mathcal{S}=(\mathcal{P}, \mathcal{L}, \mathrm{I})$ be a finite near hexagon of order $(s, t), s \geq 2$. Let $L=\left\{x_{1}, x_{2}, \ldots, x_{s+1}\right\}$ be a line of $\mathcal{S}$ and suppose $L$ is contained in precisely $k \in \mathbb{N}$ quads which we will denote 
by $Q_{1}, Q_{2}, \ldots, Q_{k}$. Put

$$
Q_{k+1}:=\left(\Gamma_{1}(L) \backslash\left(Q_{1} \cup Q_{2} \cup \cdots \cup Q_{k}\right)\right) \cup L .
$$

The sets $Q_{1}, Q_{2}, \ldots, Q_{k+1}$ determine a partition of the set $\Gamma_{1}(L)$ of points at distance 1 from $L$. If $Q_{k+1} \neq L$, then the geometry $\widetilde{Q_{k+1}}$ induced on $Q_{k+1}$ by the lines contained in $Q_{k+1}$ belongs to the family of geometries under consideration in Section 4 .

Let $p_{1}, p_{2}, \ldots, p_{v}$ with $v=|\mathcal{P}|$ be an ordering of the points of $\mathcal{S}$. Without loss of generality, we may suppose that we have ordered the points in such a way that the $(s+1) s t$ points in $\Gamma_{1}(L)$ come first. The points inside $\Gamma_{1}(L)$ will be ordered such that those contained in $Q_{1}$ come first, followed by those in $Q_{2}$, then those in $Q_{3}$, etc. The points inside each $\Gamma_{1}(L) \cap Q_{i}$ are ordered such that those in $\Gamma_{1}\left(x_{1}\right)$ come first, followed by those in $\Gamma_{1}\left(x_{2}\right)$, then those in $\Gamma_{1}\left(x_{3}\right)$, etc... As explained in Section 4 , the set $Q_{k+1} \backslash L$ can be regarded as a union of cliques of size $s$. We also assume that inside each $\Gamma_{1}\left(x_{i}\right) \cap$ $\Gamma_{1}(L) \cap Q_{k+1}$, the points are labeled such that those belonging to the same clique occupy consecutive places.

For every $i \in\{1,2, \ldots, k\}$, let $t_{2}^{(i)} \in \mathbb{N} \backslash\{0\}$ such that the generalized quadrangle $\widetilde{Q_{i}}$ has order $\left(s, t_{2}^{(i)}\right)$. Put $t_{2}^{(k+1)}:=t-t_{2}^{(1)}-t_{2}^{(2)}-\cdots-t_{2}^{(k)}$. Then for every $i \in\{1,2, \ldots, k+1\}$ and for every point $x \in L$, there are precisely $t_{2}^{(i)}$ lines through $x$ distinct from $L$ that are contained in $Q_{i}$. Put $\widetilde{k}:=k+1$ if $Q_{k+1} \neq L$ (or equivalently, $t_{2}^{(k+1)} \neq 0$ ), and $\widetilde{k}:=k$ otherwise.

For all $k_{1}, k_{2} \in \mathbb{N} \backslash\{0\}$, let $J\left(k_{1}, k_{2}\right)$ denote the real $k_{1} \times k_{2}$ matrix all whose entries are equal to 1 . For all $i, j \in\{1,2, \ldots, \widetilde{k}\}$, denote by $O^{(i, j)}$ the real zero matrix of dimensions $(s+1) s t_{2}^{(i)} \times(s+1) s t_{2}^{(j)}$, and by $E^{(i, j)}$ the real $(s+1) s t_{2}^{(i)} \times(s+1) s t_{2}^{(j)}$ block diagonal matrix whose diagonal consists of $s+1$ copies of $J\left(s t_{2}^{(i)}, s t_{2}^{(j)}\right)$, i.e. $E^{(i, j)}=\mathcal{D}\left(J\left(s t_{2}^{(i)}, s t_{2}^{(j)}\right), s+1\right)$ using the notation of Section 4.

Consider now the matrix

$$
C:=A_{2}-(s-1) A_{1}+\left(s^{2}-s+1\right) I_{v},
$$

which has $\operatorname{rank} \frac{v}{\alpha}+1$ by Lemma 2.4 . Let $\widetilde{C}\left[\widetilde{A_{2}}, \widetilde{A_{1}}\right.$, respectively $\left.\widetilde{I}\right]$ denote the submatrices obtained by selecting the first $(s+1)$ st rows and columns of $C\left[A_{2}, A_{1}\right.$, respectively $\left.I_{v}\right]$. Then

$$
\operatorname{rank}(\widetilde{C}) \leq \operatorname{rank}(C)=\frac{v}{\alpha}+1
$$

and

$$
\widetilde{C}=\widetilde{A_{2}}-(s-1) \widetilde{A_{1}}+\left(s^{2}-s+1\right) \widetilde{I}
$$

For every $i \in\{1,2, \ldots, \widetilde{k}\}$, let $R_{0}^{(i)}, R_{1}^{(i)}, R_{2}^{(i)}, R_{3}^{(i)}, R_{4}^{(i)}$ denote the relations of $Q_{i} \backslash L$ as defined in Sections 3 and 4 , and let $B_{0}^{(i)}, B_{1}^{(i)}, B_{2}^{(i)}, B_{3}^{(i)}, B_{4}^{(i)}$ denote the corresponding matrices (as defined in these sections). By Brouwer and Wilbrink [4], every two collinear points of $\Gamma_{1}(L)$ must lie in the same $Q_{i}$, and every two points of $\Gamma_{1}(L)$ at distance 2 lie 
in the same $Q_{i}$ or have the same projection on $L$. This allows to conclude that

$$
\begin{gathered}
\widetilde{A_{1}}=\left[\begin{array}{ccccc}
B_{1}^{(1)}+B_{2}^{(1)} & O^{(1,2)} & O^{(1,3)} & \cdots & O^{(1, \widetilde{k})} \\
O^{(2,1)} & B_{1}^{(2)}+B_{2}^{(2)} & O^{(2,3)} & \cdots & O^{(2, \widetilde{k})} \\
O^{(3,1)} & O^{(3,2)} & B_{1}^{(3)}+B_{2}^{(3)} & \cdots & O^{(3, \widetilde{k})} \\
\vdots & \vdots & \vdots & \ddots & \vdots \\
O^{(\widetilde{k}, 1)} & O^{(\widetilde{k}, 2)} & O^{(\widetilde{k}, 3)} & \cdots & B_{1}^{\widetilde{k}}+B_{2}^{\widetilde{k}}
\end{array}\right], \\
\widetilde{A_{2}}=\left[\begin{array}{ccccc}
B_{3}^{(1)}+B_{4}^{(1)} & E^{(1,2)} & E^{(1,3)} & \cdots & E^{(1, \widetilde{k})} \\
E^{(2,1)} & B_{3}^{(2)}+B_{4}^{(2)} & E^{(2,3)} & \cdots & E^{(2, \widetilde{k})} \\
E^{(3,1)} & E^{(3,2)} & B_{3}^{(3)}+B_{4}^{(3)} & \cdots & E^{(3, \widetilde{k})} \\
\vdots & \vdots & \vdots & \ddots & \vdots \\
E^{(\widetilde{k}, 1)} & E^{(\widetilde{k}, 2)} & E^{(\widetilde{k}, 3)} & \cdots & B_{3}^{(\widetilde{k})}+B_{4}^{(\widetilde{k})}
\end{array}\right] .
\end{gathered}
$$

Note that $E^{(i, i)}=B_{0}^{(i)}+B_{1}^{(i)}+B_{3}^{(i)}$ for every $i \in\{0,1, \ldots, \widetilde{k}\}$. We compute that

$$
\widetilde{C}:=\left[\begin{array}{ccccc}
F^{(1)} & E^{(1,2)} & E^{(1,3)} & \cdots & E^{(1, \widetilde{k})} \\
E^{(2,1)} & F^{(2)} & E^{(2,3)} & \cdots & E^{(2, \widetilde{k})} \\
E^{(3,1)} & E^{(3,2)} & F^{(3)} & \cdots & E^{(3, \widetilde{k})} \\
\vdots & \vdots & \vdots & \ddots & \vdots \\
E^{(\widetilde{k}, 1)} & E^{(\widetilde{k}, 2)} & E^{(\widetilde{k}, 3)} & \cdots & F^{(\widetilde{k})}
\end{array}\right]
$$

where $F^{(i)}=\left(B_{3}^{(i)}+B_{4}^{(i)}\right)-(s-1) \cdot\left(B_{1}^{(i)}+B_{2}^{(i)}\right)+\left(s^{2}-s+1\right) I_{(s+1) s t_{2}^{(i)}}$ for every $i \in$ $\{0,1, \ldots, \widetilde{k}\}$. We now compute the rank of the matrix $\widetilde{C}$. We will rely on the following facts which follow from Lemmas 3.4, 4.1 and the definition of the $E^{(i, j)}$ 's.

(I) The rank of the matrix $E^{(i, j)}, i, j \in\{1,2, \ldots, \widetilde{k}\}$, is equal to $s+1$.

(IIa) The rank of the matrix $F^{(i)}-E^{(i, i)}$ is equal to $\frac{s t_{2}^{(i)}\left(s^{2}-1\right)}{s+t_{2}^{(i)}}$ for every $i \in\{1,2, \ldots, k\}$.

(IIb) If $t_{2}^{(k+1)} \neq 0$, then the rank of the matrix $F^{(k+1)}-E^{(k+1, k+1)}$ is equal to $t_{2}^{(k+1)}\left(s^{2}-1\right)$.

(IIIa) The rank of the matrix $\left[F^{(i)} E^{(i, i)}\right]$ is equal to $s+1+\frac{s t_{2}^{(i)}\left(s^{2}-1\right)}{s+t_{2}^{i}}$ for every $i \in$ $\{1,2, \ldots, k\}$.

(IIIb) If $t_{2}^{(k+1)} \neq 0$, then the rank of the matrix $\left[F^{(k+1)} E^{(k+1, k+1)}\right]$ is equal to $s+1+$ $t_{2}^{(k+1)}\left(s^{2}-1\right)$.

Note that the column spaces of the matrices $E^{(i, i)}$ and $E^{(i, j)}$ coincide for all $i, j \in$ $\{1,2, \ldots, \widetilde{k}\}$ (due to the repetition of columns). We then see that the following also hold: 
(IIIa') The rank of the matrix $\left[F^{(i)} E^{(i, j)}\right]$ is equal to $s+1+\frac{s t_{2}^{(i)}\left(s^{2}-1\right)}{s+t_{2}^{i}}$ for every $i \in$ $\{1,2, \ldots, k\}$ and every $j \in\{1,2, \ldots, \widetilde{k}\}$.

(IIIb') If $t_{2}^{(k+1)} \neq 0$, then the rank of the matrix $\left[F^{(k+1)} E^{(k+1, i)}\right]$ is equal to $s+1+t_{2}^{(k+1)}\left(s^{2}-\right.$ 1) for every $i \in\{1,2, \ldots, k+1\}$.

The rank of the sum of two matrices is at most the sum of the ranks of these matrices. So, the rank of $\widetilde{C}$ is at most the sum of the ranks of the matrices

$$
\begin{gathered}
{\left[\begin{array}{ccccc}
F^{(1)}-E^{(1,1)} & O^{(1,2)} & O^{(1,3)} & \cdots & O^{(1, \widetilde{k})} \\
O^{(2,1)} & F^{(2)}-E^{(2,2)} & O^{(2,3)} & \cdots & O^{(2, \widetilde{k})} \\
O^{(3,1)} & O^{(3,2)} & F^{(3)}-E^{(3,3)} & \cdots & O^{(3, \widetilde{k})} \\
\vdots & \vdots & \vdots & \ddots & \vdots \\
O^{(\widetilde{k}, 1)} & O^{(\widetilde{k}, 2)} & O^{(\widetilde{k}, 3)} & \cdots & F^{\widetilde{k}}-E^{(\widetilde{k}, \widetilde{k})}
\end{array}\right]} \\
\\
{\left[\begin{array}{ccccc}
E^{(1,1)} & E^{(1,2)} & E^{(1,3)} & \cdots & E^{(1, \widetilde{k})} \\
E^{(2,1)} & E^{(2,2)} & E^{(2,3)} & \cdots & E^{(2, \widetilde{k})} \\
E^{(3,1)} & E^{(3,2)} & E^{(3,3)} & \cdots & E^{(3, \widetilde{k})} \\
\vdots & \vdots & \vdots & \ddots & \vdots \\
E^{(\widetilde{k}, 1)} & E^{(\widetilde{k}, 2)} & E^{(\widetilde{k}, 3)} & \cdots & E^{(\widetilde{k}, \widetilde{k})}
\end{array}\right]}
\end{gathered}
$$

i.e. at most

$$
\begin{aligned}
& \left(s^{2}-1\right) \cdot\left(\frac{s t_{2}^{(1)}}{s+t_{2}^{(1)}}+\frac{s t_{2}^{(2)}}{s+t_{2}^{(2)}}+\cdots+\frac{s t_{2}^{(k)}}{s+t_{2}^{(k)}}+t_{2}^{(k+1)}\right)+(s+1) \\
= & (s+1)+\left(s^{2}-1\right) \cdot\left(\frac{s t_{2}^{(1)}}{s+t_{2}^{(1)}}+\frac{s t_{2}^{(2)}}{s+t_{2}^{(2)}}+\cdots+\frac{s t_{2}^{(k)}}{s+t_{2}^{(k)}}+t-t_{2}^{(1)}-t_{2}^{(2)}-\cdots-t_{2}^{k}\right) \\
= & (s+1)+\left(s^{2}-1\right) \cdot\left(t-\frac{\left(t_{2}^{(1)}\right)^{2}}{s+t_{2}^{(1)}}-\frac{\left(t_{2}^{(2)}\right)^{2}}{s+t_{2}^{(2)}}-\cdots-\frac{\left(t_{2}^{(k)}\right)^{2}}{s+t_{2}^{(k)}}\right) .
\end{aligned}
$$

Now, consider $\widetilde{C}$ as a block diagonal matrix of dimensions $\widetilde{k} \times \widetilde{k}$, and denote by $\widetilde{C}(i)$ with $i \in\{1,2, \ldots, \widetilde{k}\}$ the matrix obtained from $\widetilde{C}$ by selecting the first $i$ rows (of blocks). We prove by induction on $i \in\{1,2, \ldots, k\}$ that

$$
\operatorname{rank}(\widetilde{C}(i)) \geq(s+1)+\left(\sum_{j=1}^{i} \frac{\left(s^{2}-1\right) s t_{2}^{(i)}}{s+t_{2}^{(i)}}\right) .
$$

Looking at the column span of $\widetilde{C}(1)$ and invoking (IIIa'), we indeed see that

$$
\operatorname{rank}(\widetilde{C}(1))=(s+1)+\frac{s t_{2}^{(1)}\left(s^{2}-1\right)}{s+t_{2}^{(1)}} .
$$


Now, suppose that

$$
\operatorname{rank}(\widetilde{C}(i)) \geq s+1+\sum_{j=1}^{i} \frac{s t_{2}^{(i)}\left(s^{2}-1\right)}{s+t_{2}^{(i)}}
$$

for a certain $i \in\{1,2, \ldots, k-1\}$.

For every $j \in\{1,2, \ldots, s t(s+1)\}$, let $e_{j}$ be the row matrix of dimensions $1 \times s t(t+1)$, all whose entries are equal to 0 , except for the $j$ th one which is equal to 1 . Then the real vector space $V$ of all row matrices of dimensions $1 \times s t(t+1)$ can be written as a direct sum

$$
V=V_{1} \oplus V_{2} \oplus \cdots \oplus V_{\widetilde{k}}
$$

where

$$
\begin{aligned}
V_{1} & :=\left\langle e_{1}, \ldots, e_{s t_{2}^{(1)}(s+1)}\right\rangle, \\
V_{2} & :=\left\langle e_{s t_{2}^{(1)}(s+1)+1}, \ldots, e_{s\left(t_{2}^{(1)}+t_{2}^{(2)}\right)(s+1)}\right\rangle, \\
& \vdots \\
V_{k} & :=\left\langle e_{s\left(t_{2}^{(1)}+\cdots+t_{2}^{(k-1)}\right)(s+1)+1}, \ldots, e_{s\left(t_{2}^{(1)}+\cdots+t_{2}^{(k)}\right)(s+1)}\right\rangle .
\end{aligned}
$$

If $\widetilde{k}=k+1$, i.e. $t_{2}^{(k+1)} \neq 0$, then we define

$$
V_{k+1}:=\left\langle e_{s\left(t_{2}^{(1)}+\cdots+t_{2}^{(k)}\right)(s+1)+1}, \ldots, e_{s t(s+1)}\right\rangle .
$$

For every $j \in\{1,2, \ldots, \widetilde{k}\}$, let $\Pi_{j}: V \rightarrow V_{j}$ be the canonical projection operator from $V$ on the subspace $V_{j}$. For every $j \in\{1,2, \ldots, \widetilde{k}\}$, let $W_{j}$ denote the row span of the matrix $\widetilde{C}(j)$. Then Properties (I) and (IIIa') imply the following:

- $\Pi_{i+1}\left(W_{i}\right)$ has dimension $s+1$;

- $\Pi_{i+1}\left(W_{i+1}\right)$ has dimension $s+1+\frac{s t_{2}^{(i+1)}\left(s^{2}-1\right)}{s+t_{2}^{(i+1)}}$.

It follows that $\operatorname{dim}\left(W_{i+1}\right) \geq \operatorname{dim}\left(W_{i}\right)+\frac{s t_{2}^{(i+1)}\left(s^{2}-1\right)}{t_{2}^{(i+1)}+s}=\operatorname{rank}(\widetilde{C}(i))+\frac{s t_{2}^{(i+1)}\left(s^{2}-1\right)}{t_{2}^{(i+1)}+s}$ and hence $\operatorname{rank}(\widetilde{C}(i+1))=\operatorname{dim}\left(W_{i+1}\right) \geq \operatorname{rank}(\widetilde{C}(i))+\frac{s t_{2}^{(i+1)}\left(s^{2}-1\right)}{t_{2}^{(i+1)}+s} \geq s+1+\left(\sum_{j=1}^{i+1} \frac{s t_{2}^{(j)}\left(s^{2}-1\right)}{s+t_{2}^{(j)}}\right)$.

We thus know that

$$
\operatorname{rank}(\widetilde{C}(k)) \geq s+1+\left(\sum_{j=1}^{k} \frac{s t_{2}^{(j)}\left(s^{2}-1\right)}{s+t_{2}^{(j)}}\right) .
$$

Note that $\operatorname{rank}(\widetilde{C})=\operatorname{rank}(\widetilde{C}(k))$ if $\widetilde{k}=k$.

Suppose now that $\widetilde{k}=k+1$, or equivalently, $t_{2}^{(k+1)} \neq 0$. By Properties (I) and (IIIb'), we also know 
- $\Pi_{k+1}\left(W_{k}\right)$ has dimension $s+1$;

- $\Pi_{k+1}\left(W_{k+1}\right)$ has dimension $s+1+t_{2}^{(k+1)}\left(s^{2}-1\right)$.

It follows that $\operatorname{dim}\left(W_{k+1}\right) \geq \operatorname{dim}\left(W_{k}\right)+t_{2}^{(k+1)}\left(s^{2}-1\right)$. Hence,

$\operatorname{rank}(\widetilde{C})=\operatorname{rank}(\widetilde{C}(k+1)) \geq \operatorname{rank}(\widetilde{C}(k))+t_{2}^{(k+1)}\left(s^{2}-1\right) \geq(s+1)+\left(s^{2}-1\right) \cdot\left(t_{2}^{(k+1)}+\sum_{j=1}^{k} \frac{s t_{2}^{(j)}}{s+t_{2}^{(j)}}\right)$.

Comparing the lower bound for $\operatorname{rank}(\widetilde{C})$ with the upper bound obtained in $(2)$, we find

$$
\begin{aligned}
\operatorname{rank}(\widetilde{C}) & =(s+1)+\left(s^{2}-1\right) \cdot\left(t_{2}^{(k+1)}+\sum_{j=1}^{k} \frac{s t_{2}^{(j)}}{s+t_{2}^{(j)}}\right) \\
& =(s+1)+\left(s^{2}-1\right) \cdot\left(t-\sum_{j=1}^{k} \frac{\left(t_{2}^{(j)}\right)^{2}}{s+t_{2}^{(j)}}\right),
\end{aligned}
$$

regardless of whether $\widetilde{k}=k$ or $\widetilde{k}=k+1$. From $\operatorname{rank}(\widetilde{C}) \leq \operatorname{rank}(C)$, we deduce that

$$
(s+1)+\left(s^{2}-1\right) \cdot\left(t-\sum_{j=1}^{k} \frac{\left(t_{2}^{(j)}\right)^{2}}{s+t_{2}^{(j)}}\right) \leq \frac{v}{\alpha}+1,
$$

which is equivalent to

$$
\sum_{j=1}^{k} \frac{\left(t_{2}^{(j)}\right)^{2}}{s+t_{2}^{(j)}} \geq t-\frac{s\left(s^{2}+1\right) v-s(s+1)\left(s^{2}+1\right)-s^{2} t(s+1)}{(s+1)\left(s^{4}-1\right)+s t(s-1)(s+1)^{2}+v} .
$$

Remark. In [9], the matrix $\widetilde{C}$ was written as the sum of two matrices, where one of them was the "block diagonal matrix" with "diagonal entries" equal to $F^{(1)}, F^{(2)}, \ldots, F^{(k)}$. For the case under consideration in [9], namely for regular near hexagons with parameter $t_{2}>0$, these two matrices commute and thus have a common basis of eigenvectors. For the cases under consideration here, these two matrices do not necessarily commute and so we had to resort to different methods for computing the rank of $\widetilde{C}$.

\section{Acknowledgment}

The author wants to thank Willem Haemers for discussions on the topic of this paper and for providing him with a scanned copy of the manuscript [9]. 


\section{References}

[1] J. Bamberg, J. Lansdown and M. Lee. On $m$-ovoids of regular near polygons. Des. Codes Cryptogr. 86 (2018), 997-1006.

[2] A. Bishnoi and B. De Bruyn. A new near octagon and the Suzuki tower. Electron. J. Combin. 23 (2016), Paper 2.35, 24 pp.

[3] A. Bishnoi and B. De Bruyn. The $L_{3}(4)$ near octagon. J. Algebraic Combin. 48 (2018), $157-178$

[4] A. E. Brouwer and H. A. Wilbrink. The structure of near polygons with quads. Geom. Dedicata 14 (1983), 145-176.

[5] B. De Bruyn. An introduction to Incidence Geometry. Frontiers in Mathematics. Birkhaüser, 2016.

[6] B. De Bruyn. On the adjacency algebras of near hexagons with an order. Graphs Combin. 33 (2017), 1219-1230.

[7] B. De Bruyn. On tetrahedrally closed line systems and a generalization of the Haemers-Roos inequality. Submitted.

[8] W. Haemers. Eigenvalue techniques in design and graph theory. Phd. Thesis (Eindhoven University of Technology), 1979.

[9] W. Haemers and R. Mathon. An inequality for near hexagons. Unpublished manuscript (1979).

[10] W. Haemers and C. Roos. An inequality for generalized hexagons. Geom. Dedicata 10 (1981), 219-222.

[11] D. G. Higman. Partial geometries, generalized quadrangles and strongly regular graphs. pp. 263-293 in Atti del Convegno di Geometria Combinatoria e sue Applicazioni (Univ. Perugia, Perugia, 1970). Ist. Mat., Univ. Perugia, Perugia, 1971.

[12] D. G. Higman. Invariant relations, coherent configurations and generalized polygons. pp. 27-43 in Combinatorics, Part 3: Combinatorial group theory (Proc. Advanced Study Inst., Breukelen, 1974). Math. Centre Tracts 57, Math. Centrum, Amsterdam, 1974.

[13] R. A. Horn and C. R. Johnson. Topics in matrix analysis. Cambridge University Press, 1994.

[14] R. A. Horn and C. R. Johnson. Matrix analysis. Second edition. Cambridge University Press, Cambridge, 2013. 
[15] R. Mathon. On primitive association schemes with three classes. Unpublished manuscript.

[16] A. Neumaier. Krein conditions and near polygons. J. Combin. Theory Ser. A 54 (1990), 201-209.

[17] S. E. Payne and J. A. Thas. Finite generalized quadrangles. EMS Series of Lectures in Mathematics. European Mathematical Society, 2009.

[18] E. E. Shult and A. Yanushka. Near n-gons and line systems. Geom. Dedicata 9 (1980), $1-72$.

[19] H. Van Maldeghem. Generalized polygons. Birkhäuser, 1998.

\section{Address:}

\section{Bart De Bruyn}

Department of Mathematics, Ghent University

Krijgslaan 281 (S22), B-9000 Gent, Belgium

Email: Bart.DeBruyn@Ugent.be 